\section{COALPRED: A BASIC program for computing predictions of five coalition theories}

\section{DAVID A. KRAVITZ and JOHN A. WALKER University of Kentucky, Lexington, Kentucky}

COALPRED is a computer program for computing the predictions of five descriptive theories of coalition formation: minimum resource theory (Gamson, 1961, 1964), minimum power theory (Gamson, 1964), bargaining theory (Komorita \& Chertkoff, 1973), weighted probability model (Komorita, 1974), and equal excess model (Komorita, 1979). A recent review of coalition research, including a discussion of these theories, is presented in Komorita and Kravitz (1983).

Under ideal circumstances, coalition researchers study games that maximally differentiate among contending theories, and that provide the strongest possible tests of the conceptual issues under consideration. Unfortunately, the time required to compute predictions of these theories discourages inspection of multiple games prior to selection of the game to be used. In addition, computing predictions for these theories can be confusing for those who do not know them well, and this may result in errors. For both of these reasons, researchers often use coalition games that are not ideal for their conceptual purposes. The purpose of this program (COALPRED) is to eliminate the tedium and confusion associated with computing predictions of the coalition theories listed above. This should facilitate selection of coalition games that are optimal for research purposes, and should decrease the likelihood of making incorrect predictions.

Program Input. COALPRED allows the user to specify the coalition game either in characteristic function form (by listing the minimal winning coalitions) or in weighted majority form (by specifying the quota value and the distribution of resources). The user can decide whether to consider only minimal winning coalitions or both minimal and nonminimal winning coalitions. Finally, the user can specify the maximum number of rounds of the equal excess model for which predictions will be computed (default is 20), and whether these predictions will be for reward divisions alone or both reward divisions and frequencies.

Program Output. COALPRED initially lists the assigned resources and quota value if the game was specified in weighted majority form. It then lists the winning coalitions for which predictions will be computed. This includes either minimal winning coalitions alone or both minimal and nonminimal winning coali-

The authors' mailing address is: Department of Psychology, 115 Kastle Hall, University of Kentucky, Lexington, KY 40506. tions, depending on the user's previous decision. COALPRED then prints the predictions of the five coalition theories. Both Trial 1 and asymptotic predictions are given for bargaining theory. For the equal excess model, predictions are given for each round until either the maximum number of rounds specified by the user is attained or the predictions reach an asymptote. ${ }^{1}$

Predictions of both coalition frequencies and reward divisions are given. Frequency predictions are always limited to minimal winning coalitions because all the theories predict that nonminimal winning coalitions should not occur. Rank-order frequency predictions are given for all theories except when the user has chosen to omit frequency predictions of the equal excess model, and point predictions are given for the weighted probability model. Predictions of reward division are given for all winning coalitions specified by the user. These predictions are given to two decimal places, but calculations are more precise.

Under certain conditions, the theories are unable to make predictions. When this occurs, COALPRED describes the problem and then continues with the other theories. These limitations include the following: Minimum resource theory makes no predictions when the game is given in characteristic function form. Bargaining theory and the equal excess model make no predictions when any player is included in only one minimal winning coalition. Frequency predictions cannot be made for the equal excess model on those rounds on which no coalition mutually maximizes its members' expectations. This could also occur for bargaining theory on Trial 1, but we have never encountered the problem.

COALPRED will make predictions for coalition games to which the theories may not apply. It computes predictions for veto games, although, strictly speaking, none of the theories makes predictions for such games. In nonveto games, when a player lacks a "valid" alternative to a coalition, the approach taken by the equal excess model is ambiguous (Komorita, 1979). When this situation occurs, COALPRED computes predictions in the usual manner and does not bring the issue to the user's attention. In such cases, predictions of the equal excess model may never reach an asymptote, and this appears to be most likely when the game has been given in weighted majority form.

Limitations. COALPRED is limited to simple coalition games: It will not make predictions for multivalued games. It will deal with groups of size three and above. However, COALPRED is limited to approximately 80 total coalitions (minimal and nonminimal winning). In large groups there may be many nonminimal winning coalitions, and if the user requests predictions for these coalitions, the program may not work. Running time varies with the game, the machine, and the printer. It can be extremely slow when the game includes more 
than six players and/or more than about 15 coalitions. This problem could be eliminated by revising the program for use on a mainframe computer.

Documentation. COALPRED is accompanied by extensive documentation describing both the program and the theories dealt with by the proram.

Language and Computer. COALPRED was written in BASIC and was developed on an Apple IIt microcomputer. Brief suggestions for extensions to other versions of BASIC are given in the documentation.

Availability. Listings of COALPRED and accompanying documentation can be obtained without charge from: David A. Kravitz, Department of Psychology, 115 Kastle Hall, University of Kentucky, Lexington, KY 40506. A floppy disk containing the program can be obtained from David Kravitz at cost.

\section{REFERENCES}

Gamson, W. A. (1961). A theory of coalition formation. American Sociological Review, 26, 373-382.

Gamson, W. A. (1964). Experimental studies of coalition formation. In L. Berkowitz (Ed.), Advances in experimental social psychology (Vol. 1, pp. 81-110). New York: Academic Press.

KomoritA, S. S. (1974). A weighted probability model of coalition formation. Psychological Review, 81, 242-256.

Komorita, S. S. (1979). An equal excess model of coalition formation. Behavioral Science, 24, 369-381.

Komorita, S. S., \& Chertroff, J. M. (1973). A bargaining theory of coalition formation. Psychological Review, 80, 149-162.

Komorita, S. S., \& Kravitz, D. A. (1983). Coalition formation: A social psychological approach. In P. B. Paulus (Ed.), Basic group processes (pp. 179-203). New York: Springer-Verlag.

Roth, A. E. (1977a). Bargaining ability, the utility of playing a game, and models of coalition formation. Journal of Mathematical Psychology, 16, 153-160.

Roth, A. E. (1977b). The Shapley value as a von NeumannMorgenstern utility. Econometrica, 45, 657-664.

Shaptex, L. S. (1953). A value for n-person games. In H. W. Kuhn \& A. W. Tucker (Eds.), Contributions to the theory of games (Vol. 2, pp. 307-317). Princeton, NJ: Princeton University Press.

Shapley, L. S., \& Shubik, M. (1954). A method for evaluating the distribution of power in a committee system. American Political Science Review, 48, 787-792.

\section{NOTE}

1. Payoff predictions of the Roth-Shapley value (Roth, 1977a, 1977b; Shapley, 1953; Shapley \& Shubik, 1954) are not explicitly made by COALPRED. However, if the user requests predictions for nonminimal winning coalitions, minimum power theory's prediction of reward division in the grand coalition is equal to the payoff prediction of the Roth-Shapley value. If the user does not request predictions for nonminimal winning coalitions, Roth-Shapley payoff predictions can be calculated from the pivotal power values that are printed. Payoff predictions of the weighted probability model also can be computed from the predictions of reward division and frequencies.

(Manuscript accepted for publication March 9, 1984.) 